\title{
Upper GI bleed after surgery for cyanotic heart disease
}

\author{
Nirmal Kumar • Saket Aggarwal • \\ Deepak Kumar Satsangi
}

Received: 14 February 2012 / Accepted: 8 September 2012 /Published online: 11 December 2012

(C) Indian Association of Cardiovascular-Thoracic Surgeons 2012

We describe a rare case of massive Upper Gastrointestinal bleed (UGI) bleed following a Bidirectional Glenn (BDG) operation. This patient was a 10 year old girl who presented with complaint of cyanosis since early childhood. Echocardiography revealed the diagnosis of complete unbalanced Atrioventricular (AV) canal defect with severe valvular and infundubular pulmonary stenosis with a left superior vena cava. After cardiac catheterisation, she underwent bilateral BD Glenn operation. Post-operatively, her recovery was uneventful. She was discharged on post-op day 5 on digoxin, aspirin and lasilactone.

One and a half months later she presented again with right pleural effusion. She did not have signs of raised jugular venous pressure, ascites or hepatomegaly. A chest tube was inserted which drained chlylous fluid, which was confirmed on biochemical analysis. Her hemogram was remarkable for severe thrombocytosis with platelet count of $19,00,000 / \mu 1$ and mild leucocytosis; her haemoglobin, bleeding time, clotting time \& prothrombin time were within normal limits. She was initially managed conservatively with fat free diet, medium chain fatty acid supplementation and Octreotide infusion. Echocardiography revealed normal bilateral Glenn flow. However, her chylothorax persisted and surgical intervention was sought. She underwent thoracotomy with lymphatic ligation and fibrin glue application at the chylous leak side, after which she was recovering well. Six days later she developed massive UGI bleeding and collapsed. Her $\mathrm{Hb}$ was $3.7 \mathrm{~g} / \mathrm{dl}$. An UGI endoscopy was planned, but could not be carried out as she continued to bleed profusely and was critically ill. Despite resuscitation, she died the same day.

Bleeding complications are common in patients with cyanotic heart diseases [1]. They are attributed to relative

N. Kumar $(\bowtie) \cdot$ S. Aggarwal $\cdot$ D. K. Satsangi

Department of CTVS, GB Pant Hospital,

New Delhi-2, India

e-mail: nirmalk20@gmail.com polycythemia, shortened platelet survival and alteration in clotting factors. Paradoxically there is tendency for thrombotic complications also due to erythrocytosis and increased haematocrit, altered platelet aggregation etc. Earlier reports in literature of UGI bleed in patients with cyanotic congenital heart disease suggest the probable causes to be esophageal varices following splenic vein thrombosis or rarely due to GI stromal tumors. In our case, the former is more likely as the increased viscosity from polycythemia and thrombocytosis could have led to splenic vein thrombosis resulting in esophageal varices and subsequently UGI bleed. The other possibility of non-steroidal anti-inflammatory drug (NSAID) induced UGI hemorrhage could not be ruled out. Kejariwal et al. reported a case of undiagnosed Tetralogy of Fallot (TOF) in a 48 year old lady presenting with variceal bleed due to splenic vein thrombosis [2, 3]. Mahajan et al. reported Gastro-Intestinal Stromal Tumor (GIST) as a cause for UGI bleeding in TOF patient while other rarely reported causes include angiodysplasia $[4,5]$.

\section{References}

1. Kurt M, Litmathe J, Roehrborn A, Feindt P, Boeken U, Gams E. Abdominal complications following open-heart surgery: a report of 12 cases and review of the literature. Acta Cardiol. 2006;61:301-6.

2. Kejariwal D, Sarkar N, Roy S, Bhattacharya M. Fallot's tetralogy presenting with variceal bleed. J Postgrad Med. 2001;47:194-5.

3. Miller TL, Lang P, Liberthson R, Grillo HC, Israel EJ. Upper gastrointestinal haemorrhage as a late complication of congenital heart disease. J Pediatr Gastroenterol Nutr. 1996;23:452-6.

4. Mahajan N, Kondur A, Afonso L. Gastrointestinal bleeding in tetralogy of fallot. Oman Med J. 2008;23:187-8.

5. Varma P, Misra M, Radhakrishnan VV, Neelakandhan KS. Fatal post-operative gastro intestinal hemorrhage because of angiodysplasia of small intestine in aortic regurgitation. Interact Cardiovasc Thorac Surg. 2004;3:118-20. 